\title{
Análisis de la logística de reparto a domicilio de la compra de alimentación y hogar. Aplicación a Consum en el área de Valencia. Propuestas para su mejora.
}

\author{
María Rosa Arroyo López \\ Dpto. Ingeniería e Infraestructura de los Transportes, \\ Universitat Politècnica de València \\ José Vicente Colomer Ferrándiz \\ Dpto. Ingeniería e Infraestructura de los Transportes, \\ Universitat Politècnica de València
}

\section{RESUMEN}

El problema de la distribución urbana en las grandes ciudades y en particular, el reparto de mercancías a domicilio, no tiene una solución simple, ya que confluyen múltiples factores y diversos actores con intereses diferentes. El objetivo de este artículo es analizar las principales características de este servicio, muy poco estudiado dentro de la logística urbana, con el fin de proponer medidas de actuación, que garanticen la sostenibilidad desde los puntos de vista económico, social y ambiental. El análisis se aplica a la empresa Consum, que se estudia con mayor detalle y para finalizar se proponen 29 medidas de actuación para este caso práctico.

\section{INTRODUCCIÓN}

Según datos del Libro Blanco del E-commerce del 2015 [1], de cada $100 €$ de facturación en comercio electrónico, $40 €$ corresponden a artículos físicos, y que por lo tanto requieren ser transportados hasta llegar a su destino final en la cadena de suministro, el consumidor final. Hoy día, resulta natural adquirir un producto que se encuentra a disposición del cliente a unos metros de su domicilio por vía electrónica. Este cambio de mentalidad está generando grandes flujos de transporte de productos a domicilio, algunas empresas trasladan el coste del envío al cliente, otras lo asumen como herramienta para fidelizarlos.

El ritmo natural diario de la sociedad, con largas jornadas de trabajo y pasando poco tiempo en sus hogares, contrasta con la creciente demanda de envío a domicilio. Por este motivo, los repartos fallidos rondan el $25 \%$ del total, problema que junto a la logística inversa incrementa notablemente los costes de la operación. A este problema se plantean soluciones de reparto no atendido, como buzones de recepción, consignas, recogida en establecimientos y similares.

La logística de distribución a domicilio de alimentación y hogar presenta además particularidades asociadas al alto volumen y peso de la mercancía, además de tratarse de 
productos perecederos y de alta rotación que precisan de un transporte rápido y ágil.

Como resultado, la demanda de transporte de mercancías a domicilio aumenta cada día, con el consiguiente crecimiento de las externalidades asociadas al tráfico de vehículos comerciales en las ciudades y que lleva al planteamiento de la sostenibilidad a largo plazo de la gestión actual de la logística urbana a nivel económico, social y ambiental.

\section{METODOLOGÍA}

En primer lugar, se estudia la oferta y la operativa del reparto a domicilio en distintas empresas, según datos públicos, obtenidos a través de webs, llamadas telefónicas y visitas a establecimientos.

Seguidamente se analiza la situación detallada de Consum, mediante la información directa obtenida a través de entrevistas con SD Logística (empresa que opera el reparto a domicilio de Consum) y con el área logística de Consum.

A partir de lo anterior, con los conocimientos obtenidos, se puede abordar un diagnóstico de la situación que permite establecer las principales ventajas e inconvenientes según los distintos agentes implicados y establecer un DAFO.

Detectadas las principales debilidades y amenazas y teniendo en cuenta las fortalezas y oportunidades existentes, se plantean distintas alternativas de actuación utilizando un Análisis Multicriterio teniendo en cuenta criterios económicos, sociales y ambientales, así como la visión de los agentes implicados.

Con todo ello, se señalan las mejores alternativas obtenidas para la mejora de la logística del reparto a domicilio de la compra de alimentación y hogar de la empresa Consum.

\section{EL REPARTO A DOMICILIO DE LA COMPRA DE ALIMENTACIÓN Y HOGAR}

\section{1 Características generales}

Con el fin de entender las particularidades del reparto a domicilio de la compra de alimentación y hogar, se realiza un análisis cualitativo basado en visitas a tiendas, entrevistas telefónicas y datos públicos de las webs de las empresas que ofrecen estos servicios. La recogida de datos se centra en las características de los productos, hábitos de consumo y ubicación de los establecimientos que condicionan el modelo de distribución.

Con todo ello se obtiene que las principales características del reparto a domicilio de la compra de alimentación y hogar son las siguientes:

- Frecuencia de servicio elevada. La compra de alimentación se caracteriza por su entrega en un plazo máximo de 24horas. La caducidad de los productos transportados es el elemento diferenciador respecto a otros bienes de consumo. 
- Oferta de franjas horarias. A diferencia del transporte a domicilio de otros productos no alientarios, donde el comprador elige recibir su pedido en amplias franjas de horarios, (4-6 horas), en el sector de alimentación y hogar, sí es común la garantía de entrega por franjas, de en torno a 2 horas. Esta característica se comparte con otros tipos de transporte especializados como ocurre con productos voluminosos como muebles.

- Baja capacidad de almacenamiento por parte del consumidor.

- Productos de alta rotación.

- Logística inversa. Los retornos se realizan en vacío y dadas las características del servicio resulta complicado encontrar solución a esta cuestión. Se utilizan cajas y recipientes reutilizables y no se entregan embalajes al consumidor.

- Pedidos de volumen y peso considerable. La compra de alimentación y hogar y en especial cuando se solicita el servicio a domicilio, se compone de artículos de peso y volumen considerable, como bebidas, productos de limpieza, etc.

- Gran variabilidad de productos. Son muy diversos y por tanto, los requisitos de transporte también lo son: congelados, frescos, embotellados de cristal, otros productos frágiles, etc.

- Picking de cajas intensivo. Las unidades básicas de almacenaje utilizadas para el transporte a domicilio son cajas de fácil apilamiento y acoplamiento, algunas de ellas isotermas y cajones abiertos. Al coexistir productos muy diversos, de diferentes tamaños, formas, pesos y requerimientos resulta complicado estandarizar los medios de transporte y almacenaje.

○ Comportamiento del consumidor. Este tipo de compra es susceptible de convertirse en periódica, no se encuentran estudios al respecto, y según las entrevistas realizadas parece ser que existen perfiles de consumidores que demandan los servicios con cierta periodicidad, en las tiendas de proximidad principalmente. Otros sin embargo corresponden a compras esporádicas.

- Kilometraje. Las distancias recorridas son reducidas en comercios de proximidad debido al gran número de establecimientos existentes en las zonas residenciales, en el caso de hipermercados pueden darse distancias algo mayores. No obstante, ambos acotan las áreas de reparto.

- Carga. Se realiza en los almacenes de los establecimientos o parkings, aunque no suele ser posible en ciudad disponer de muelles interiores para la recepción de mercancías, los vehículos utilizados en el reparto a domicilio son de menores dimensiones y sí que pueden entrar.

- Descarga en la calle. La descarga se realiza principalmente en el viario, utilizando aparcamientos convencionales y zonas de cargas y descarga. Así mismo, se observan estacionamientos indebidos en aceras, pasos de peatones y doble fila. 
- Lugar de entrega. Dadas las características de los productos, el lugar de entrega es el interior del domicilio particular, no siendo comunes otras tipologías como transporte a pie de calle o entrega asíncrona.

○ No se comparten vehículos con otros cargadores.

- Flota de vehículos, propia o en régimen de renting utilizada en exclusividad para el servicio.

○ No se utilizan gestores de rutas automáticos y la planificación de entregas es principalmente manual.

\section{2 Análisis de la oferta}

El Ministerio de Agricultura, Alimentación y Medio Ambiente, elabora Estudios de Mercado a través del Observatorio de Consumo y Distribución Alimentaria y establece un Índice de Calidad de Servicio. De esta manera, obtiene variables cualitativas y cuantitativas sobre los hábitos de consumo de la población y tendencias de la distribución de los productos alimentarios. Para ello, se realizan 400 visitas Mystery Shopper en las que se evalúan diversos aspectos de las instalaciones y atención al cliente.

Respecto al servicio de reparto, se pregunta la posibilidad de envío a domicilio, por lo que se tiene el porcentaje de comercios que ofrecen este servicio según el tipo de establecimiento. En la tabla 1, se muestran los datos de las últimas encuestas realizadas, se servirán simplemente de referencia por no disponer de estudios más actualizados

\begin{tabular}{lcccc}
\hline & $\mathbf{2 0 0 7}$ & $\mathbf{2 0 0 8}$ & $\mathbf{2 0 1 0}$ & $\mathbf{2 0 1 1}$ \\
\hline Tienda tradicional & 11,3 & 8 & 22,4 & 16 \\
Mercados & 20 & 20 & 35,5 & 24 \\
Tienda descuento & 4 & 10 & 1,3 & 4 \\
Autoservicios/Superservicios & 64 & 60 & 68,4 & 64 \\
Supermercados $\left(\mathbf{4 0 0}-\mathbf{9 9 9} \mathbf{~ m}^{2}\right)$ & 82 & 94 & 87,2 & 84 \\
Supermercados $\left(\mathbf{1 0 0 0}-\mathbf{2 4 9 9} \mathbf{~ m}^{2}\right)$ & 92 & 96 & 95,2 & 100 \\
\hline
\end{tabular}

Tabla 1. Porcentaje de comercios que ofrecen el envío a domicilio. Fuente MAGRAMA.

Teniendo en cuenta el dato más reciente, del 2011, se observa que de los supermercados de 
mayor tamaño que han sido entrevistados, todos ofrecen la posibilidad de reparto a domicilio. Si se consideran los supermercados medianos, esta cifra disminuye hasta un $84 \%$ y en el caso de pequeños supermercados o autoservicios hasta el $64 \%$.

Los hipermercados también rozan casi la totalidad, con un porcentaje del $96 \%$. No ocurre lo mismo con los formatos comerciales más reducidos, como mercados o tiendas tradicionales o de conveniencia con una oferta mucho menor.

En el caso de la Comunidad Valenciana, se estudian las principales empresas del sector y se clasifican según precio del servicio y características de la oferta (tabla 2).

\begin{tabular}{|c|c|c|c|c|c|c|c|c|}
\hline & Domicilio & Variables & $\begin{array}{l}\text { Gratis a } \\
\text { partir de }\end{array}$ & $\begin{array}{c}\text { Horas } \\
\text { reparto }\end{array}$ & $\begin{array}{c}\text { Total } \\
\text { semana }\end{array}$ & Franjas & Online & Recogida \\
\hline Consum & $3 €$ & & $\begin{array}{c}60 € / 75 € \\
\text { si socio }\end{array}$ & $\begin{array}{c}\text { 8h/día x } \\
5+4\end{array}$ & 44 & 2 & no & no \\
\hline Mercadona & $4,21 €$ & & no & $\begin{array}{c}8 \mathrm{~h} / \text { día } \mathrm{x} \\
6 \mathrm{~d}\end{array}$ & 48 & 2 & $7,21 €$ & no \\
\hline Alcampo & $\begin{array}{c}>6,90 € \\
(\mathrm{~km})\end{array}$ & $\mathrm{Km}$ & no & $\begin{array}{c}\text { 13h/día } \\
\text { x 6d }\end{array}$ & 78 & 3 & $9,90 €$ & no \\
\hline Aldi & no & & & & & & no & no \\
\hline Carrefour & 5 a $7 €$ & Zonas & no & $\begin{array}{c}\text { 12h/día } \\
\text { x } 6 \mathrm{~d}\end{array}$ & 72 & 2 & $\begin{array}{l}6>90 € \\
\text { si no } 9\end{array}$ & $3 €$ \\
\hline Día & $3 €$ & Compra & & & & & no & no \\
\hline Eroski & & & & & & & no & \\
\hline $\begin{array}{l}\text { El Corte } \\
\text { Inglés }\end{array}$ & $8 €$ & & $\begin{array}{c}120 \\
€ / 100 € \\
\text { tarjeta ECI }\end{array}$ & 12h/día & & 4 & $8 €$ & \\
\hline Lidl & no & & & & & & no & \\
\hline Covirán & & & & & & & no & \\
\hline $\begin{array}{l}\text { Grupo } \\
\text { Miquel }\end{array}$ & & & & & & & no & \\
\hline Vidal & no & & & & & & no & \\
\hline Simply & & & & & & & no & \\
\hline Unide & & & & & & & no & \\
\hline Mas y Mas & $2,40 €$ & & 60 & $\begin{array}{c}\text { Mañana/ } \\
\text { tarde }\end{array}$ & 48 & & no & \\
\hline Musgrave & & & & & & & & \\
\hline
\end{tabular}

Tabla 2. Principales tiendas del sector de la alimentación y hogar y características del envío a domicilio. Elaboración propia. 
Del análisis anterior se pueden extraer las siguientes conclusiones:

\subsubsection{Comercio electrónico/ venta telefónica}

Para analizar el reparto a domicilio, se debe tener en cuenta la posibilidad de compra online, puesto que condiciona la prestación del servicio.

\subsubsection{Procesamiento de pedidos}

A la vista de los datos, y sin conocer la gestión interna del servicio, no parece que ninguna empresa disponga de almacén de procesamiento de pedidos para el cliente.

\subsubsection{Expediciones en hipermercados}

En los hipermercados se distribuyen también productos de distintas gamas, hecho que condiciona las expediciones, y da lugar a diferentes esquemas: desde integración total a sistemas de pedido independientes según el circuito.

\subsubsection{Horarios y slots}

Las ventanas horarias de reparto oscilan entre 2 y 4 horas. En cuanto a la amplitud de horarios ofertados, varían entre 44 y 78 horas semanales. El tiempo máximo de entrega sí que parece seguir la misma línea en todos los supermercados, en torno a 24 horas.

\subsubsection{Precios}

La variable principal que condiciona el coste del envío es el importe de la compra en la mayoría de los casos, aunque hay otros modelos como: precio fijo o variable por kilometraje o zona.

\subsubsection{Tipos de gestión del transporte}

Las empresas de distribución de compra de alimentación y hogar pueden optar por externalizar el proceso de reparto a domicilio u operarlo ellas mismas con sus propios medios. Según el grado de subcontratación y las operaciones que se externalizan se puede clasificar como: gestión directa integral, gestión directa con subcontratación del transporte y externalización completa.

\subsection{Comparación con otros modelos}

Con el propósito de poder comparar con otros posibles modelos más avanzados, se observan las características de grandes proveedores como Walmart o Tesco. Las principales diferencias halladas son:

- Amplios horarios de entorno 15 horas al día sin interrupción, que cubren la demanda fuera del horario laboral habitual.

- Slots más reducidos.

- Sistemas automáticos de comunicación con el cliente.

○ Funcionamiento de tienda online independiente de las físicas.

- Almacenes de procesamiento de pedidos mecanizados.

○ Tarifas en función del slot. 


\section{ANÁLISIS DEL REPARTO A DOMICILIO DE LA EMPRESA CONSUM}

Consum es una cooperativa, integrada por socios trabajadores y consumidores, que desarrolla su actividad en el sector de la distribución comercial, a través de los supermercados Consum, Consum Basic y las franquicias Charter. Es la mayor cooperativa del arco mediterráneo y una de las primeras empresas en el sector de la distribución en España., formada por una red comercial de más de 638 supermercados.

La gestión del reparto a domicilio se encuentra externalizada de manera integral y la realiza la empresa SD Logística. La operativa del servicio atiende a criterios organizativos históricos, y se realiza de manera manual.

El área de reparto es de máximo $8 \mathrm{~km}$ y $3 \mathrm{~km}$ en el caso de capitales de provincia y se garantiza la entrega dentro de franjas de 2 horas, que el cliente elige al pasar por caja, de lunes a viernes en horario de mañana y tarde y el sábado por la mañana. La mayoría de tiendas ofrecen este servicio, dentro de Valencia capital sólo el 10\% de las tiendas no están adheridas.

Existe también la posibilidad de realizar el pedido por teléfono, en cuyo caso se paga un recargo por el servicio de preparación del pedido.

Las tarifas establecidas son distintas para algunos colectivos: socios-clientes y pensionistas, siendo como máximo de $6 €$ y gratuitas en cualquier caso a partir de $60 €)$.

Los horarios de reparto no son iguales para todas las tiendas y la demanda previsible se calcula con estimaciones de históricos. Cada repartidor tiene asignado un vehículo del cual se hace responsable de su mantenimiento básico: limpieza, repostaje, inspecciones técnicas, revisiones, etc. No obstante, cada Consum no tiene por qué tener asignado un repartidor.

Según los estudios de Consum, el tipo de cliente que demanda el servicio de reparto de la compra a domicilio es habitual y no es común que clientes ocasionales lo soliciten. Por este motivo, no disponen de sistemas de organización de reparto en tiempo real y atienden a demandas históricas.

Consum garantiza a sus clientes la entrega a domicilio de la compra dentro de franjas de 2 horas. El procedimiento de asignación de horarios es manual y no existe ningún tipo de gestión de rutas ni planificación previa.

\subsection{Diagnóstico de logística de reparto a domicilio}

Con el fin de entender las diferentes perspectivas se realiza un análisis DAFO para los distintos agentes implicados: SD Logística, Consum, el cliente y la sociedad en su conjunto. 


\section{DEBILIDADES}

- Rigidez del sistema de reparto

- Tipo de entrega atendida

- Mercancía de alimentación y hogar: los perecederos requieren agilidad en al transporte.

- Compromiso con Consum de cumplimiento de objetivos.

- Entrega en franjas horarias de 2 horas: poca flexibilidad a las condiciones de circulación.

\section{AMENAZAS}

- Gran competencia en el sector.

- Alto coste unitario de cada reparto.

- Dificultad de automatización de procesos.

- Operadores logísticos con gran especialización.

- Normativas municipales heterogéneas.

- Limitaciones de entrada de vehículos comerciales en muchas ciudades

\section{FORTALEZAS}

- Especialización en reparto a domicilio.

- Relación cercana y comunicación directa con Consum.

- Utilización de las instalaciones de Consum: supermercados, espacio en parking y oficinas.

- Contrato con Consum cerrado. No hay incertidumbre en recursos necesarios.

\section{OPORTUNIDADES}

- Nuevas modalidades de entrega emergentes.

- Resultados positivos en las experiencias con vehículos eléctricos.

- Sistemas de optimización de rutas y programas de gestión.

\section{Análisis DAFO de Consum.}

\section{DEBILIDADES}

- Paga un alto coste unitario por pedido.

- No recupera los costes.

- El transporte supone un volumen importante en su actividad: con la externalización completa pierde poder de supervisión y toma de decisiones.

\section{AMENAZAS}

- Aumento de la demanda del servicio, que puede hacerlo insostenible en la situación actual.

- Sistema de reparto y gestión de la información obsoleto y sin relación con sus "socios".

- Sostenibilidad del reparto a domicilio a algunos colectivos.

\section{FORTALEZAS}

- Aumento de la demanda del servicio, que puede hacerlo insostenible en la situación actual.

- Sistema de reparto y gestión de la información obsoleto y sin relación con sus "socios".

- Sostenibilidad del reparto a domicilio a algunos colectivos.

\section{OPORTUNIDADES}

- Nuevos operadores logísticos especializados.

- Nuevos sistemas de gestión de información y comunicación.

- Posibilidades de nuevas opciones de envío.

- Posibilidad de comercio electrónico. 


\begin{tabular}{|c|c|}
\hline $\begin{array}{l}\text { DEBILIDADES } \\
-\end{array}$ & $\begin{array}{l}\text { AMENAZAS } \\
\text { - Sostenibilidad del servicio a largo plazo en las } \\
\text { mismas condiciones }\end{array}$ \\
\hline $\begin{array}{l}\text { FORTALEZAS } \\
\text { - } \quad \text { Precio bajo del servicio comparado con la } \\
\text { competencia } \\
\text { - } \quad \text { Compra gratuita a partir de un importe } \\
\text { relativamente bajo } \\
\text { - Ventajas para algunos colectivos } \\
\text { - } \quad \text { Garantía de recepción del pedido en franjas de } \\
\text { - } 2 \text { horas } \\
\text { Beneficios derivados de la condición de socio }\end{array}$ & $\begin{array}{l}\text { OPORTUNIDADES } \\
\text { - } \quad \text { Posibilidad de nuevas modalidades de entrega } \\
\text { - } \quad \text { Posibilidad de comercio electrónico }\end{array}$ \\
\hline
\end{tabular}

Análisis DAFO de la sociedad.

\section{DEBILIDADES}

- Congestiones y tráfico.

- Contaminación

- Ruido

- Utilización del viario y vehículos comerciales mal estacionados.

\section{FORTALEZAS}

- Una buena gestión del sistema de transporte que optimice los flujos, reduce los desplazamientos de particulares en vehículo privado.

\section{AMENAZAS}

- Crecimiento de externalidades del transporte

\section{OPORTUNIDADES}

- Vehículos sostenibles: nuevos combustibles. Ya existen pruebas con vehículos eléctricos.

Tabla 3. DAFO según agente implicado. Elaboración propia.

\subsection{Propuestas de actuación}

Dada la complejidad del sistema de reparto a domicilio, debido a la gran casuística que se presenta y a la multitud de agentes implicados, se ha optado por realizar un análisis multicriterio, evaluando las diferentes actuaciones desde los puntos de vista de la sostenibilidad económica, social y ambiental.

Esta información se ha organizado en fichas, con el fin de tener de una manera ordenada las implicaciones de los distintos agentes según los tipos de sostenibilidad descritos y se han evaluado las afecciones tomando el rango de valores de -3 a 3 . 


\begin{tabular}{|c|c|c|}
\hline ACTUACIÓN A1 & \multicolumn{2}{|l|}{ Permiso para parar en doble fila de 10 minutos } \\
\hline TIPO & \multicolumn{2}{|l|}{ Administrativa } \\
\hline SUBTIPO & \multicolumn{2}{|l|}{ Estacionamiento } \\
\hline DESCRIPCIÓN & \multicolumn{2}{|c|}{$\begin{array}{l}\text { Permiso otorgado por las administraciones locales a los operadores logísticos que realizan servicios de reparto a } \\
\text { domicilio para parar un máximo de } 10 \text { minutos en doble fila, siempre que no interfiera el tráfico y no exista una plaza } \\
\text { de carga y descarga a menos de } 150 \text { metros. }\end{array}$} \\
\hline \multicolumn{3}{|c|}{ Sostenibilidad económica } \\
\hline ACTORES & AFECCIÓN & VALORACIÓN \\
\hline CONSUM & $\begin{array}{l}\text { Según las condiciones actuales del contrato no le afectaría a corto plazo. Sin embargo, en } \\
\text { la renovación podrían acordar con SD Logística una reducción de los } \\
\text { vehículos/conductores contratados por disminuir el tiempo medio de la entegra. }\end{array}$ & 2 \\
\hline SD LOGÍSTICA & $\begin{array}{l}\text { Disminución del tiempo medio de entrega por facilitarse el aparcamiento, que se traduce } \\
\text { en ahorro económico. Con esta medida el estacionamiento sería en muchos casos } \\
\text { inmediato en el portal del cliente. }\end{array}$ & 3 \\
\hline CLIENTES & $\begin{array}{l}\text { El cliente paga el mismo precio y según las condiciones actuales no afectaría. Sin } \\
\text { embargo, un aumento de la eficiencia en el servicio y disminución de tiempos podría } \\
\text { traducirse en una mejor oferta. }\end{array}$ & 1 \\
\hline SOCIEDAD & Sin influencia. & 0 \\
\hline \multicolumn{3}{|c|}{ Sostenibilidad ambiental } \\
\hline ACTORES & 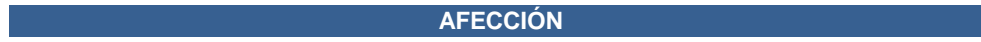 & VALORACIÓN \\
\hline CONSUM & $\begin{array}{l}\text { Mejora de la sostenibilidad ambiental en la medida en que Consum es el cargador aunque } \\
\text { no opere el transporte. }\end{array}$ & 2 \\
\hline SD LOGÍSTICA & $\begin{array}{l}\text { Menor contaminación de los vehículos asociada a una reducción de los kilómetros } \\
\text { recorridos por búsqueda de aparcamiento. }\end{array}$ & 2 \\
\hline CLIENTES & Sin afección & 0 \\
\hline SOCIEDAD & Mejora de la calidad del aire, por reducción de emisiones. & 3 \\
\hline \multicolumn{3}{|c|}{ Sostenibilidad social } \\
\hline ACTORES & AFECCIÓN & VALORACIÓN \\
\hline CONSUM & Reducción de vehículos con la marca Consum mal estacionados. & 1 \\
\hline SD LOGÍSTICA & Facilita el trabajo de los repartidores. & 2 \\
\hline CLIENTES & Perciben una mejora en el servicio. & 1 \\
\hline SOCIEDAD & Incremento del número de vehículos comerciales parados en doble fila. & -2 \\
\hline
\end{tabular}

\section{Figura 1. Ejemplo de ficha de valoración. Elaboración propia.}


Finalmente se obtienen 29 posibles actuaciones:

Alternativas relacionadas con la administración

\begin{tabular}{cc}
\hline Código & Nombre \\
\hline A1 & Permiso para parar en doble fila de 10 minutos \\
A2 & Reserva electrónica de plazas de carga y descarga \\
A3 & Incremento de la vigilancia en plazas de carga y descarga \\
A4 & Implantación de carril multiusos \\
A5 & Plazas de carga y descarga destinadas exclusivamente a operadores de transporte de mercancías \\
\hline
\end{tabular}

Alternativas relacionadas con el servicio.

\begin{tabular}{|c|c|c|}
\hline Código & Subtipo & Nombre \\
\hline S1 & Conducción & Formación destinada a conductores \\
\hline $\mathbf{S 2}, \mathbf{S 3}$ & Descarga & Aparcamiento en parking público o privado \\
\hline S4 & Carga & Reserva de espacio en parking de Consum para el vehículo. \\
\hline S5 & Horarios & Distribución nocturna \\
\hline S6 & Horarios & Tarifa con discriminación por franjas horarias \\
\hline S7 & Horarios & Selección de todas las franjas posibles para el cliente \\
\hline S8 & Horarios & Establecer horarios alternos en tiendas próximas \\
\hline S9 & Procesamiento & Plataforma logística para procesamiento de pedidos \\
\hline S10 & Comunicación & Mejora de la comunicación interna \\
\hline S11 & Rutas & Software de gestión de rutas \\
\hline
\end{tabular}

Alternativas relacionadas con los vehículos.

\begin{tabular}{cc}
\hline Código & Nombre \\
\hline V1 & Vehículos eléctricos \\
V3 & Optimización del tamaño de los vehículos \\
\hline Código & Alternativas relacionadas con la gestión. \\
\hline G1 & Nombre \\
\hline
\end{tabular}




\begin{tabular}{cc}
\hline G2 & Gestión directa y subcontratación de transporte a uno o varios operadores \\
G3 & Externalización completa: pago por reparto \\
\hline Alternativas relacionadas con los puntos de entrega. \\
\hline Código & Nombre \\
\hline E1 & Servicio de entrega "a pie de calle" \\
E3 & Servicio de recogida en tienda \\
E4 & Tienda online \\
E5 & Entrega en puntos de recogida \\
E6 & Adquisición en una tienda y recogida en otra. \\
\hline
\end{tabular}

Alternativas relacionadas con la organización.

Código Nombre

01

Bases para un nuevo esquema organizativo

\section{CONCLUSIONES}

Muchas empresas han utilizado hasta el momento el envío gratuito como estrategia de marketing para captar clientes asumiendo los costes de la operación. Según declaraciones del Director del Logística de Consum, solamente el 19\% de las compras (las consideradas "de despensa", con importe superior a $100 €$ ) pueden soportar el coste del servicio.

A la vista de los datos, surgen reflexiones a futuro sobre la sostenibilidad a largo plazo de la actual logística urbana y la compatibilidad con las nuevas tendencias sociales. La aplicación de medidas encaminadas a la mitigación de las externalidades del transporte y la racionalización en el uso de espacios en las ciudades resulta fundamental para garantizar la sostenibilidad económica, social y ambiental en el futuro.

\section{REFERENCIAS}

[1] Observatorio Ecommerce. (2015). Libro Blanco de la Logística en Ecommerce

[2] Campayo Rodríguez, C. (2002). Los supermercados y su entorno en las distintas zonas urbanas. Distribución y Consumo, 61-74.

[3] Cerdá Suárez, L. M. (2002). Tipología y evolución de los centros comerciales. Distribución y Consumo, 43-58. 
[4] Colomer Ferrándiz, J. V., Coca Castaño, P., Díaz y Pérez de la Lastra, J. M., Insa Franco, R., \& Sánchez-Barcaiztegui Moltó, V. El transporte terrestre de mercancías: organización y gestión. Valencia: Fundación Instituto Portuario de Estudios y Cooperación.

[5] Gevaers, R., Van de Voorde, E., \& Vanelslander, T. (2009). Characteristics of innovations in last mile logistics - using best practices, case studies and making the link with green and sustainable logistics. Association for European Transport and Contributors

[6] Institut Cerdà (2010). Logística urbana

[7] Ministerio de Fomento. (2013). Estrategia logística en España. Madrid.

[8] Antún, J. P. (2010). Distribución Urbana de Mercancías. Estrategias con Centros Logísticos.

[9] Oficina de Comercio y Territorio. Pateco (2013). Informe Anual de la Distribución Urbana Minorista. Comunidad Valenciana.

[10] Planteamientos Estratégicos para la Logística Urbana: Perspectiva de la Relación Universidad-Estado-Empresa. (2013). INGENIARE, Universidad Libre-Barranquilla (15), 133-141.

[11] Reagan, A., Park, M. (2009). Issues in emerging home delivey operations.

[12] Sanz Marzá, G. (2009). Innovación en la distribución urbana de mercancías. Caso práctico: supermercados Caprabo. III International Conference on Industrial Engineering and Industrial Management, (págs. 2025-2033). Barcelona-Terrasa.

[13] Sanz Marzá, G., \& Pastor Moreno, R. (2008). Metodología para la definición de un sistema logístico que trate de lograr una distribución urbana de mercancías eficiente. XII Congreso de Ingeniería de Organización. Burgos.

[14]Toribio, J. J., Díaz-Giménez, J., Campos, R., \& Gómez Bengoechea, G. (2012). La cadena agroalimentaria en España. Madrid: IESE Business School. 